\title{
Computational investigation of the adsorption of aspartic acid on $\mathrm{CaCO}_{3}$ vaterite
}

\author{
ALICIA SCHUITEMAKER ${ }^{1,2,3}$, KATARZYNA B \\ KOZIARA $^{1}$, PAOLO RAITERI ${ }^{1,2,3}$, JULIAN D GALE ${ }^{1,2,3}$ \\ AND RAFFAELLA DEMICHELIS ${ }^{1,2,3}$ \\ ${ }^{1}$ Curtin University \\ ${ }^{2}$ Curtin Institute for Computation \\ ${ }^{3}$ The Institute for Geoscience Research (TIGeR) \\ Presenting Author: alicia.schuitemaker@curtin.edu.au
}

Vaterite, the least stable crystalline polymorph of anhydrous calcium carbonate $(\mathrm{CaCO} 3)$ at ambient conditions, is attracting increasing attention due to its occurrence in living organisms. [13]

Due to vaterite being predominantly found in biological environments, biomolecules are believed to be important for its stabilization and growth. For example, L- and D-chiral acidic amino acids have been recently used to grow left- and righthanded vaterite toroids, respectively.[4] Additional investigations have shown that the formation of homochiral clusters of aspartic acid at the surface may be responsible for directing the growth of these toroids.[5]

This study aims to understand the atomic details of the interaction between vaterite and aspartic acid. Classical molecular and lattice dynamics were employed to identify stable vaterite surfaces and to explore the structure and dynamics of water on these surfaces. Enhanced sampling methods were then applied to study the adsorption of L- and D-aspartic acid and its zwitterion on vaterite (Figure 1).

[1] Lenaz et al. (2006), Eur J Mineral 18, 143-158.

[2] Wightman, Wallis \& Aston (2018), Flora 241, 27-34.

[3] Nehrke et al. (2012), Geochem Geophys Geosyst 13, 75-8.

[4] Jiang et al. (2017), Nat Comms 8, 15066-8.

[5] Jiang et al. (2019), Nat Comms 10, 2318-9.

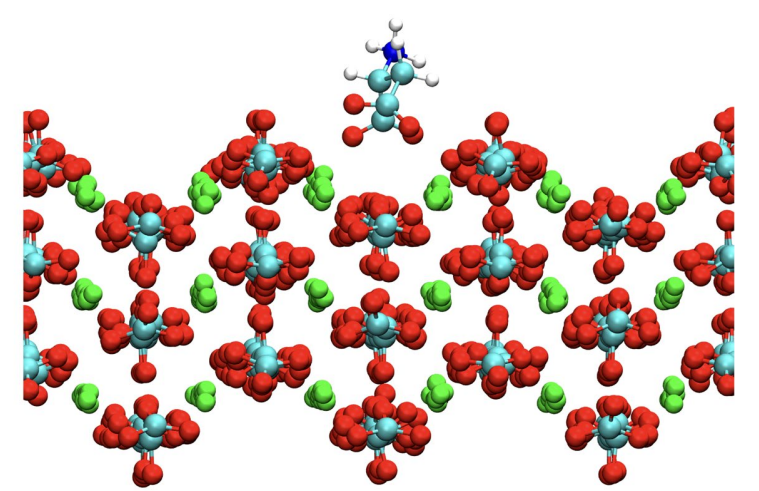

Figure 1: D-Aspartic acid zwitterion binding to a vaterite surface. Calcium, carbon, oxygen, nitrogen and hydrogen are shown in green, cyan, red, blue and white, respectively. For clarity water is not depicted. 